\title{
Iatrogenic seeding of skull base chordoma following endoscopic endonasal surgery
}

\author{
*David T. Fernandes Cabral, MD,1 Georgios A. Zenonos, MD,1 Juan C. Fernandez-Miranda, MD,1 \\ Eric W. Wang, MD, ${ }^{2}$ and Paul A. Gardner, MD'
}

Departments of ${ }^{1}$ Neurological Surgery and ${ }^{2}$ Otolaryngology, University of Pittsburgh School of Medicine, Pittsburgh, Pennsylvania

OBJECTIVE latrogenic tumor seeding after open surgery for chordoma has been well described in the literature. The incidence and particularities related to endoscopic endonasal surgery (EES) have not been defined.

METHODS The authors retrospectively reviewed their experience with EES for clival chordoma, focusing on cases with iatrogenic seeding. The clinical, radiographic, pathological, and molecular characterization data were reviewed.

RESULTS Among 173 EESs performed for clival chordomas at the authors' institution between April 2003 and May 2016, 2 cases complicated by iatrogenic seeding (incidence 1.15\%) were identified. The first case was a 10-year-old boy, who presented 21 months after an EES for a multiply recurrent clival chordoma with a recurrence along the left inferior turbinate, distinct from a right petrous apex recurrence. Both appeared as a T2-hypertintense, T1-isointense, and heterogeneously enhancing lesion on MRI. Resection of the inferior turbinate recurrence and debulking of the petrous recurrence were both performed via a purely endoscopic endonasal approach. Unfortunately, the child died 2 years later due progression of disease at the primary site, but with no sign of progression at the seeded site. The second patient was a 79-year-old man with an MRI-incompatible pacemaker who presented 19 months after EES for his clival chordoma with a mass involving the floor of the left nasal cavity that was causing an oro-antral fistula. On CT imaging, this appeared as a homogeneously contrast-enhancing mass eroding the hard palate inferiorly, the nasal septum superiorly, and the nasal process of the maxilla, with extension into the subcutaneous tissue. This was also treated endoscopically (combined transnasal-transoral approach) with resection of the mass, and repair of the fistula by using a palatal and left lateral wall rotational flap. Adjuvant hypofractionated stereotactic CyberKnife radiotherapy was administered using 35 Gy in 5 fractions. No recurrence was appreciated endoscopically or on imaging at the patient's last follow-up, 12 months after this last procedure. In both cases, pathological investigation of the original tumors revealed a fairly aggressive biology with 1 p36 deletions, and high Ki-67 levels (10\%-15\%, and > 20\%, respectively). The procedures were performed by a team of right-handed surgeons (otolaryngology and neurosurgery), using a 4-handed technique (in which the endoscope and suction are typically passed through the right nostril, and other instruments are passed through the left nostril without visualization).

CONCLUSIONS Although uncommon, iatrogenic seeding occurs during EES for clival chordomas, probably because of decreased visualization during tumor removal combined with mucosal trauma and exposure of subepithelial elements (either inadvertently or because of mucosal flaps). In addition, tumors with more aggressive biology (1p36 deletions, elevated Ki-67, or both) are probably at a higher risk and require increased vigilance on surveillance imaging and endoscopy. Further prospective studies are warranted to evaluate the authors' proposed strategies for decreasing the incidence of iatrogenic seeding after EES for chordomas.

https://thejns.org/doi/abs/10.3171/2017.6.JNS17111

KEY WORDS clival chordoma; iatrogenic seeding; oncology; skull base; endoscopic endonasal approach

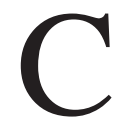
HORDOMAS are primary bone tumors arising from vestigial notochordal remnants along the cephalocaudal axis, from the clivus to the sacrum. ${ }^{15,16}$ Generally, they are slow-growing tumors, representing $1 \%-4 \%$ of all primary bone malignancies and $0.2 \%$ of all intracranial neoplasms. ${ }^{13,19}$
Although chordomas are considered low-grade malignancies, they are locally aggressive, with an incidence of local recurrence ranging between $20 \%$ and $49 \%$ of cases. ${ }^{5,8,10,13,17}$ Distal metastases, such as lung, skin, liver, bone, and lymph nodes, have been reported in 10\%-43\% of patients. ${ }^{9}$ Some factors that have been associated with

ABBREVIATIONS EES $=$ endoscopic endonasal surgery.

SUBMITTED January 11, 2017. ACCEPTED June 19, 2017.

INCLUDE WHEN CITING Published online December 22, 2017; DOI: 10.3171/2017.6.JNS17111.

* Drs. Fernandes Cabral and Zenonos contributed equally to this study. 
a higher risk of distal metastases are young age, atypical features on histopathological studies, and sacrococcygeal or vertebral tumor localization. ${ }^{3}$

Iatrogenic seeding during resection or biopsy procedures in these tumors is well described, and has been reported to occur in $2.8 \%-7.3 \%$ of cases. ${ }^{3,11}$ In all these cases, surgical pathway seeding presented as a hyperintense lesion along the surgical corridor on T2-weighted MRI. Fischbein et al. ${ }^{9}$ have proposed that a distance $>2.5-3.0$ $\mathrm{cm}$ from the tumor bed would be suggestive of surgical pathway seeding. This complication is not approach dependent, but despite an extensive review of the literature, we could not identify any reports on the incidence of iatrogenic seeding following endoscopic endonasal surgery (EES) for resection of skull base chordomas. In this report, we review our experience with 173 EESs for clival chordomas, focusing on cases in which we observed iatrogenic seeding. We discuss the patterns of seeding as well as the potential reasons explaining these patterns. Based on our findings, we have made recommendations that may prevent this complication.

\section{Methods}

We retrospectively reviewed records for 173 EESs for skull base chordoma performed at our institution between April 2003 and May 2016. All cases had at least 6 months of clinical and radiographic follow-up. Additionally, we performed an extensive review of the literature using PubMed, MEDLINE, and Google Scholar. For our search, we used the key words "clival chordoma," "skull base chordoma," "seeding," and "recurrence." Studies with iatrogenic seeding of skull base chordoma were reviewed. Generally, all patients undergoing EES for chordomas are followed by both ENT and neurosurgery practitioners on every clinic follow-up visit, and receive an endoscopic assessment in addition to dedicated skull base MRI (which includes thin-cut contrast-enhanced spoiled gradient-recalled acquisition sequences, as well as thincut T2-weighted images). For patients who cannot have MRI, contrast-enhanced CT scans are performed. All imaging does include the nasal passages and is reviewed by a neuroradiologist or head and neck radiologist.

Endoscopic endonasal evaluation is performed for a minimum of 3 months postoperatively, but typically continues for at least 6 months to ensure complete healing. The routine timing of follow-up after surgery is as follows: immediately postoperatively, 3 months, 9 months, and then yearly thereafter unless there are radiographic or clinical concerns. For patients from other states or countries, after the first 3-6 months postoperatively, follow-up is performed through correspondence with the patient as well as imaging, which is sent to us for review at our comprehensive skull base conference consisting of neurosurgeons, ENT specialists, oculoplastic surgeons, and neuroradiologists with specialization in skull base pathology. Additionally, every attempt is made to ensure endoscopic follow-up of these patients, with care given by experienced ENT colleagues who are more accessible to them. The MRI studies in all 173 patients were always routinely and prospectively evaluated for seeding in the surgical tract,
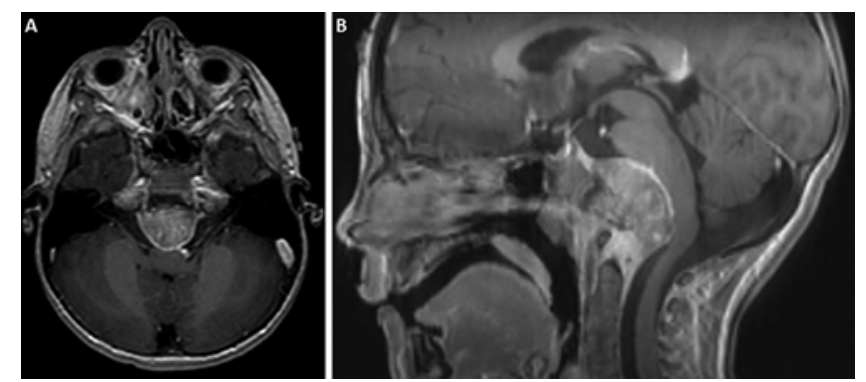

FIG. 1. Admission T1-weighted MR images obtained with contrast materials showing local recurrence of chordoma at the craniocervical junction following multiple prior resections at other institutions, as well as experimental chemotherapy. A and B: Axial and sagittal images, respectively, showing severe compression of the brainstem by the tumor.

and were re-reviewed at the time of data collection for the study. This study was performed with the approval of the institutional review board of the University of Pittsburgh.

\section{Results}

In our retrospective study, we were able to identify 2 cases of $173(1.15 \%)$ EESs for skull base chordoma in which iatrogenic seeding occurred. Both involved recurrences in the left nasal cavity. The mean follow-up was 39 months (range 6-148 months), and 137 of the remaining 171 cases without evidence of seeding had at least 19 months of follow-up (the shortest time frame in which iatrogenic seeding was observed in this series). Four patients were ultimately lost to follow-up, but these patients had at least 36 months of follow-up.

\section{Case Presentations}

The first case occurred in a 10-year-old boy who underwent multiple prior resections as well as experimental chemotherapy and proton beam radiotherapy before EES for a large recurrent clival chordoma at our institution (Fig. 1). A left vascularized inferior turbinate flap was used for reconstruction because most of the nasal septum had been removed during prior surgeries. Pathological investigation reflected a relatively aggressive tumor biology with $1 \mathrm{p} 36$ deletions, and a Ki-67 level of 10\%-15\% (no 9p/p16 deletion was detected). There were areas of classic, chondroid, and solid forms, as well as foci of poorly differentiated tumor. Approximately 21 months after his resection, the patient developed a recurrence along the left inferior turbinate with extension to the maxillary sinus causing obstruction of the nasal passage. This was seen as a hyperintense lesion on T2-weighted MRI (Fig. 2). He also developed recurrence in the skull base causing facial weakness. This was treated with palliative repeat EES for resection of the tumor, as well as palliative debulking of the recurrent tumor involving the skull base. Repeat radiation therapy was discussed but ultimately was not deemed feasible, given the amount of radiation he had received previously. Notably, the Ki-67 level from this recurrent tumor was as high as $25 \%$ focally (increased from the original tumor). Unfortunately, the child went on to have multiple subsequent recurrences that ultimately led to his death 2 years later, but he had no evidence of recurrence at the site of seeding. 

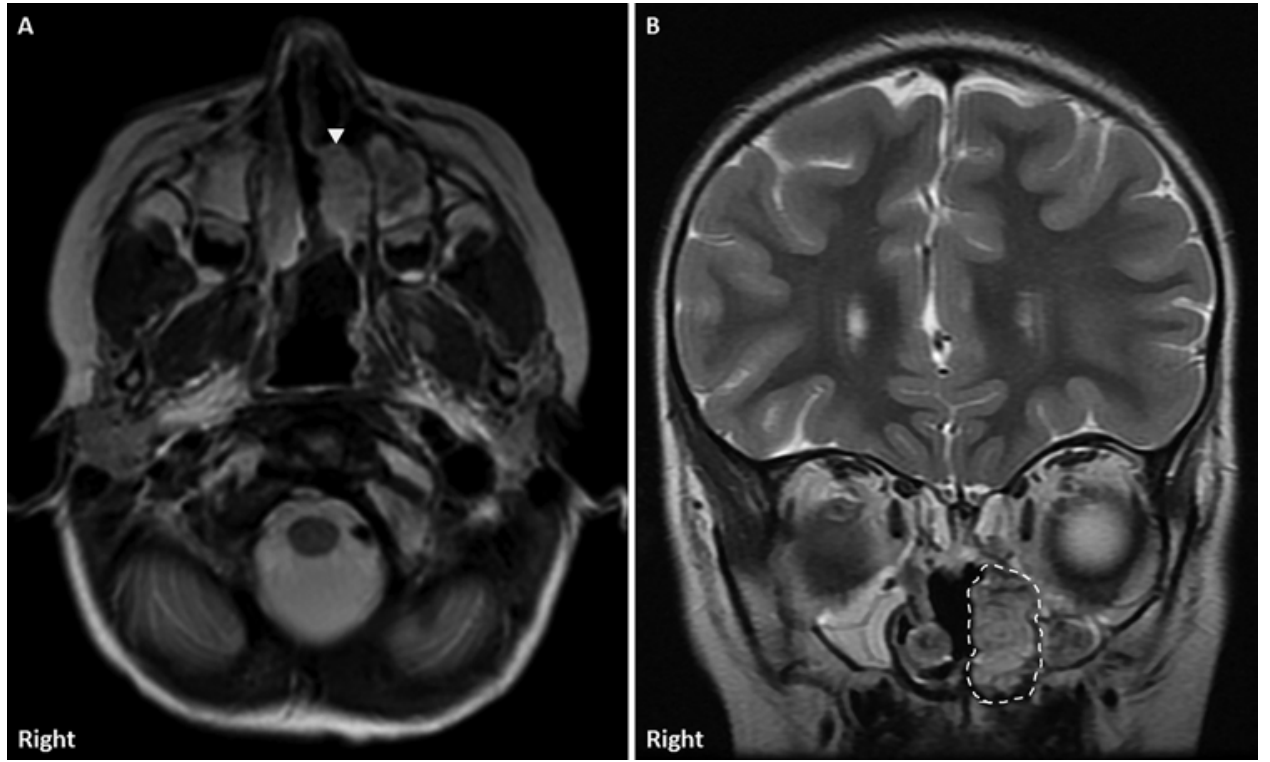

FIG. 2. Postoperative follow-up imaging obtained at 21 months after the surgery showing recurrence in the left inferior turbinate. A and B: Axial and coronal T2-weighted MR images, respectively, showing T2-hyperintense tumor seeding along the left inferior turbinate (white arrowhead and white dashed line).

The second case was a 79-year-old man who underwent EES for resection of an extensive clival chordoma (Fig. 3). This patient had undergone a biopsy at another institution prior to presentation, which resulted in right-sided blindness. Interestingly, the patient originally received the diagnosis of sarcoma, given the atypical features of the tumor. Because of the patient's advanced age and the complete encasement of both carotid arteries, a near-total resection was performed $(90 \%-95 \%)$, followed by hypofractionated CyberKnife stereotactic radiotherapy (4 fractions). A left nasal-septal flap was used for reconstruction. As in the previous case, the patient's original pathological findings reflected an atypical tumor with $1 \mathrm{p} 36$ deletions and a Ki-67 level of $>20 \%-25 \%$ in certain areas (no 9p/p16 deletion was detected). The "atypical" designation was given because, although the tumor largely retained a classic chordoma morphology, there were some areas of solid epithelial growth, increased nuclear atypia, and multiple mitoses. Surveillance imaging was performed with contrasted CT scans only, due to an MRI-incompatible pacemaker. Nineteen months postoperatively, there was no tumor growth within the original resection bed, but there was a contrast-enhancing mass in the floor of the left nasal cavity creating an oro-antral fistula (Fig. 4). This was also evident on endoscopy, and after a biopsy in the clinic, the mass was confirmed to represent chordoma. This was treated with a combined endoscopic endonasal-transoral resection and repair of the fistula with a palatal flap. Adjuvant CyberKnife hypofractionated radiotherapy (35 Gy in 5 fractions) was administered in the resection bed. At his last follow-up, 12 months later, there was no evidence of any recurrence.

Both surgeries were performed using a 4-handed technique, and all surgeons were right-handed. In this 4-handed technique, the endoscope usually occupies the right superior nostril, the suction the right inferior nostril, and dissecting instruments (such as scissors, pituitary rongeurs, and cup forceps) are passed through the left nostril with the neurosurgeon's right hand. Of note, the nasal septum, when intact, hides the surgical corridor through the left nostril, and therefore the instruments are passed blindly.

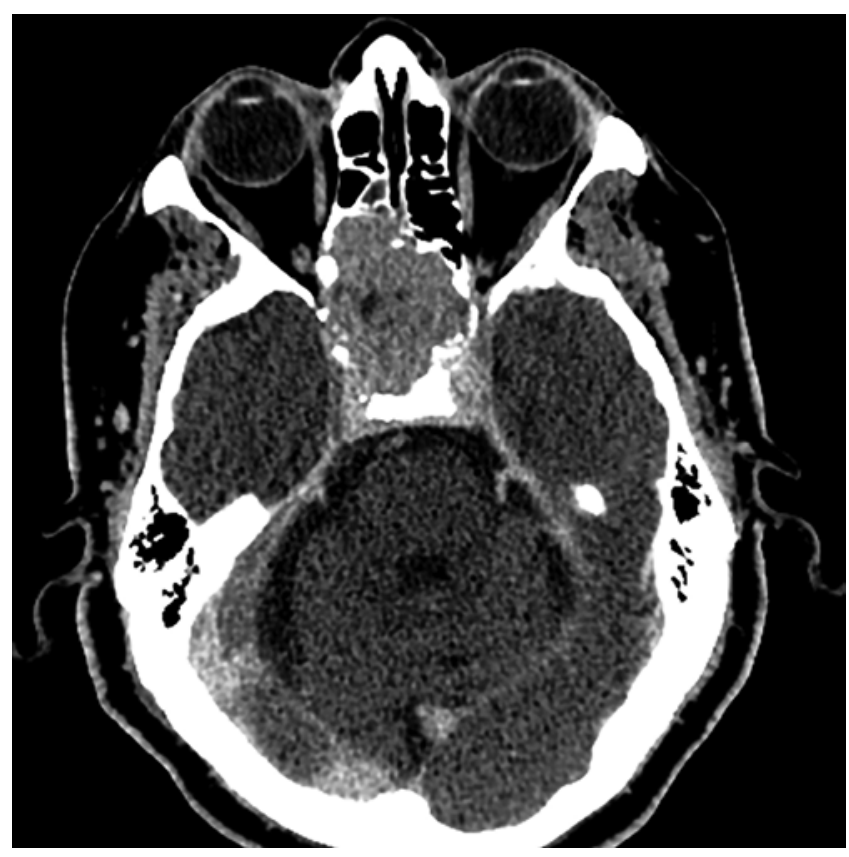

FIG. 3. Preoperative axial CT obtained with contrast showing an enhancing mass centered within the anterior skull base and nasopharynx, with erosion of the clivus and extension into the sphenoid and cavernous sinuses. 

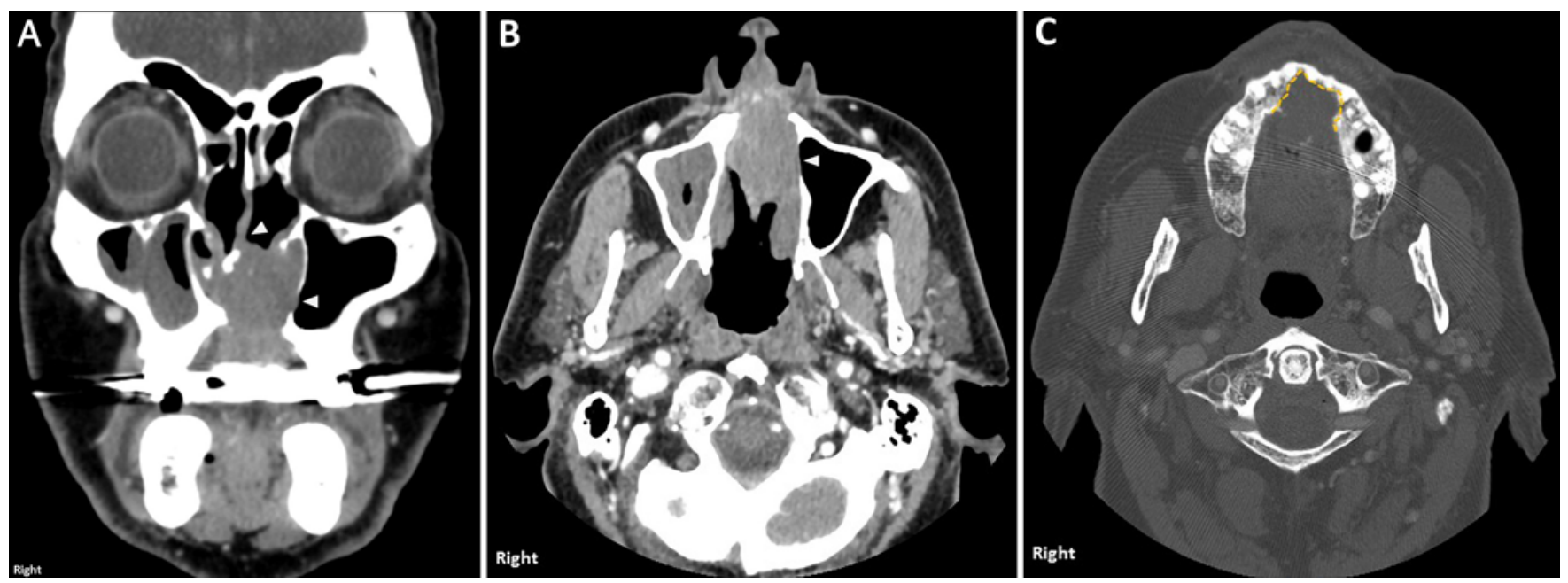

FIG. 4. Postoperative contrast CT head scans obtained 2 years after surgery. A and B: Coronal and axial slides showing a softtissue mass emanating from the floor of the left nasal cavity and eroding the nasal septum, the medial wall of the left maxillary sinus (white arrowheads), and the hard palate. C: Axial bone window cut showing the hard palate erosion (yellow dashed line).

\section{Molecular Characteristics in Our Series}

In 100 of 173 cases we found $1 \mathrm{p} 36$ on fluorescence in situ hybridization, and in $70 \%$ of these tumors $>15 \%$ of their cells were harboring $1 \mathrm{p} 36$ deletions. Therefore, the incidence of iatrogenic seeding within this group was $2.9 \%$ ( 2 of 70$)$. Of the 144 tumors for which the Ki-67 was available, 27 had a Ki-67 level $>10 \%$. The incidence of iatrogenic seeding within this group was $7.4 \%(2 / 27)$. Eighteen patients had Ki-67 levels > 10\%, as well as 1p36 deletions in $>15 \%$ of their tumor cells, and 2 of these 18 patients $(11.1 \%)$ had iatrogenic seeding.

\section{Literature Review}

Our literature review failed to identify any other cases of iatrogenic seeding after endoscopic endonasal approaches for resection of skull base chordomas. There are, however, reports of seeding following other anterior approaches, including sublabial transsphenoidal and microscopic transnasal approaches. These reports do not allow for an accurate prediction of incidence. Table 1 summarizes important findings from our literature review.

\section{Discussion}

\section{Tumor Biology}

Iatrogenic seeding of neoplastic cells during biopsy or resection is a well-known phenomenon that has been reported for a wide variety of malignancies. ${ }^{4,6,7,14}$ For a neoplastic cell to invade and grow in a different tissue, several complex steps have to take place. The neoplastic cells must be able to evade the natural immune protective mechanisms of the host, have specific surface molecules able to interact with the host's normal cells, and be able to induce neovascularity to support their growth., ${ }^{1,2,12,18}$ Although the specific mechanisms involved in chordoma have not been elucidated, it has been hypothesized that local vasodilation, release of cytokines, and microtrauma during surgery might play a role. ${ }^{11}$ Notably, seeding of a chordoma on an intact epithelial or mucosal surface has never been reported.

Interestingly, both of our cases represented dedifferentiated tumors with 1p36 deletions and high Ki-67 levels. It is not known if this is a requirement for iatrogenic seeding of chordomas, but certainly such tumors with worrisome pathological features warrant increased vigilance for seeding on surveillance imaging as well as endoscopy. Notably, at least in our series, patients with 1p36 deletions, elevated Ki-67 (> 10\%), or both had progressively higher incidences of seeding $(2.4 \%, 7.9 \%$, and $11.1 \%$, respectively) than the overall incidence in this cohort (1.15\%).

\section{Incidence of Seeding}

The incidence of surgical pathway seeding for clival chordomas following open procedures has been reported to range between $2.8 \%$ and $7.3 \%$.,11 Given that an en bloc resection is not feasible with EES (as opposed to surgeries for spinal chordomas), one would theoretically expect piecemeal resection to be associated with an increased risk for seeding. Instead, the incidence of iatrogenic seeding in our series is lower than that for either open spine or skull base approaches (1.15\%). The reasons behind this lower incidence are not entirely clear. A plausible explanation is that an intact nasal epithelium may constitute an unfavorable milieu for tumor cell implantation when compared with an incised or traumatized tissue surface with exposed submucosal and/or mesenchymal elements, which is the case in most open skull base and spinal surgical procedures. Of course, mucosal trauma from the passage of instruments during endoscopic endonasal approaches may result in focal breaches of the epithelium, increasing the chances of seeding. Furthermore, the use of flaps for reconstruction (nasal-septal flaps, or lateral nasal wall flap) also leads to exposure of submucosal and/or mesenchymal elements, which could be seeded with tumor. However, the overall exposure of these submucosal and/or mesenchymal elements during EES is probably 


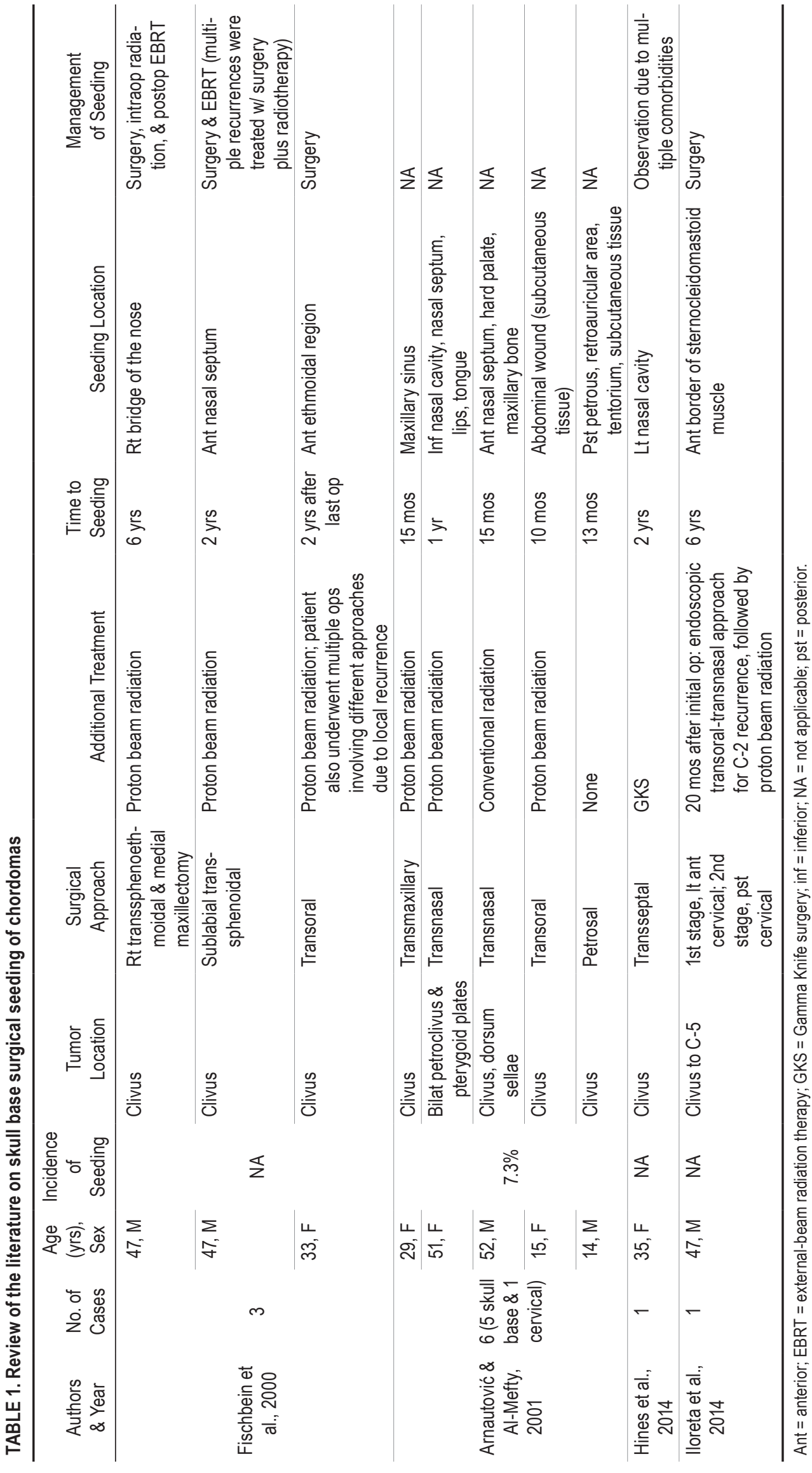


smaller than that with open procedures. Improved visualization of dropped fragments afforded by the endoscope may also be a factor.

\section{Patterns of Recurrence}

As briefly discussed earlier, both of our iatrogenic seeding cases had a recurrence in the left nasal cavity. The most likely explanation for this pattern of recurrence is that with the 4-handed technique, the instruments used to remove the tumor pass through the left nostril without visualization by the endoscope. Although chordomas are usually soft tumors and can be removed with suction alone, larger pieces of tumor or pieces of bone infiltrated with tumor are also usually removed through the left nostril with instruments (e.g., pituitary rongeurs or cup forceps). During removal, any contact of the tumor pieces with the nasal wall is blind. Furthermore, because the tumor pieces are usually very friable, it is possible that small tumor pieces may be dropped during the extraction from the left nasal passage. If these tumor fragments are not detected immediately, continued blind passage of instruments through the same nostril will result in plastering the nasal cavity with tumor cells, increasing the chances of contact with submucosal elements. Without frequent irrigation, small fragments of tumor may become adherent. In addition, the use of mucosal flaps from the left nasal cavity in both of our cases probably increased the surface area of exposed submucosal elements and the likelihood of contact with tumor cells, and consequently the risk for seeding.

\section{Measures That Could Potentially Decrease the Risk for latrogenic Seeding}

Some surgical strategies have been proposed in the literature to decrease the risk of surgical pathway seeding in nonendoscopic procedures. Arnautović and Al-Mefty suggest that once the approach is completed and before tumor removal, the walls of the operative tunnel should be coated with fibrin glue and large cotton patties; then, once the tumor has been debulked, removal of the cotton patties with the fibrin glue should be followed by placement of a fat graft. They also suggest that before closing, all the contaminated drapes, instruments, and gloves should be replaced by a new clean set, a statement also supported by Iloreta et al. 3,11

With EES, a conscious effort to fully visualize each fragment as it is removed by transferring the endoscope to the left nostril can minimize tumor contact with the nasal wall, as well as help early detection of any dropped pieces of tumor so they can be removed immediately. An effort to remove smaller pieces with a larger rongeur (which can completely encompass the fragment) at each pass may also help decrease tumor contact with the nasal wall as well as decrease the inadvertent fragmentation of the tumor during the extraction. Taking care to minimize denudation of the nasal mucosal lining is also important, so as to decrease the exposure of submucosal and/or mesenchymal elements. At our institution, we now use commercially available nasal sleeves to minimize trauma to the nasal mucosa, but also to prevent direct contact of the tumor with the nasal cavity. In addition, at the end of the procedure, the nasal cavity should be carefully inspected for tumor fragments, and copious irrigation should be used, not only in the tumor bed, but also in the nasal cavity.

The exposed subepithelial elements of the mucosal flap donor sites that are used for reconstruction of skull base defects probably increase the risk for seeding. For this reason, whenever possible, flaps should be placed on the side contralateral to tumor removal or the exposed tissues should be protected with a barrier during tumor removal.

It is also important to ensure that no tumor is dropped during the passage of the tumor fragments to the scrub technicians, so as to prevent contamination of other surgical instruments, drapes, and gloves. Once tumor removal is complete, both the surgeons and the scrub technicians should change their outer gloves, and another set of instruments should be used for the final irrigation and reconstruction.

During follow-up imaging and endoscopy, there should be increased vigilance for seeding, especially for tumors with worrisome features on pathological investigation. Given the low incidence of tumor seeding with EES, inclusion of the operative corridor in the adjuvant radiation plan is not recommended. However, this could be considered for tumors with extremely aggressive histological types, especially for cases with extensive demucosalization. Isolated deposits of tumor seeding can be effectively managed with additional surgery.

\section{Conclusions}

Although uncommon, iatrogenic seeding occurs during EES for clival chordoma, probably because of decreased visualization during tumor removal combined with mucosal trauma and exposure of subepithelial elements (either inadvertently or because of mucosal flaps). In addition, tumors with more aggressive biology (1p36 deletions, high Ki-67 levels, or both) are probably at a higher risk and require increased vigilance on surveillance imaging and endoscopy. Further prospective studies are warranted to evaluate our proposed strategies for decreasing the incidence of iatrogenic seeding after endoscopic endonasal procedures for chordomas. The low incidence of tumor seeding with EES does not support the routine inclusion of the nasal cavity in the postoperative radiation therapy plan.

\section{References}

1. Abbas AK, Lichtman AH, Pillai S: Cellular and Molecular Immunology. Philadelphia: Elsevier, 2014

2. Al-Tameemi M, Chaplain M, d'Onofrio A: Evasion of tumours from the control of the immune system: consequences of brief encounters. Biol Direct 7:31, 2012

3. Arnautović KI, Al-Mefty O: Surgical seeding of chordomas. J Neurosurg 95:798-803, 2001

4. Barloon TJ, Yuh WT, Sato Y, Sickels WJ: Frontal lobe implantation of craniopharyngioma by repeated needle aspirations. AJNR Am J Neuroradiol 9:406-407, 1988

5. Chibbaro S, Cornelius JF, Froelich S, Tigan L, Kehrli P, Debry $\mathrm{C}$, et al: Endoscopic endonasal approach in the management of skull base chordomas-clinical experience on a large series, technique, outcome, and pitfalls. Neurosurg Rev 37:217-225, 2014

6. Cole GW Jr, Sindelar WF: Iatrogenic transplantation of osteosarcoma. South Med J 88:485-488, 1995 
7. Curran AJ, Smyth D, Kane B, Toner M, Timon CI: Exfoliated malignant cells in glove and instrument washings following head and neck surgery. Clin Otolaryngol Allied Sci 21:281283, 1996

8. Di Maio S, Rostomily R, Sekhar LN: Current surgical outcomes for cranial base chordomas: cohort study of 95 patients. Neurosurgery 70:1355-1360, 2012

9. Fischbein NJ, Kaplan MJ, Holliday RA, Dillon WP: Recurrence of clival chordoma along the surgical pathway. AJNR Am J Neuroradiol 21:578-583, 2000

10. Hines JP, Ashmead MG, Stringer SP: Clival chordoma of the nasal septum secondary to surgical pathway seeding. Am J Otolaryngol 35:431-434, 2014

11. Iloreta AM, Nyquist GG, Friedel M, Farrell C, Rosen MR, Evans JJ: Surgical pathway seeding of clivo-cervical chordomas. J Neurol Surg Rep 75:e246-e250, 2014

12. Kindt TJ, Goldsby RA, Osborne BA: Kuby Immunology. New York: W. H. Freeman, 2007

13. Koutourousiou M, Gardner PA, Tormenti MJ, Henry SL, Stefko ST, Kassam AB, et al: Endoscopic endonasal approach for resection of cranial base chordomas: outcomes and learning curve. Neurosurgery 71:614-625, 2012

14. Paolucci V, Schaeff B, Schneider M, Gutt C: Tumor seeding following laparoscopy: international survey. World J Surg 23:989-997, 1999

15. Salisbury JR: [Embryology and pathology of the human notochord.] Ann Pathol 21:479-488, 2001 (Fr)

16. Salisbury JR: The pathology of the human notochord. J Pathol 171:253-255, 1993

17. Sen C, Triana AI, Berglind N, Godbold J, Shrivastava RK: Clival chordomas: clinical management, results, and complications in 71 patients. J Neurosurg 113:1059-1071, 2010
18. Töpfer K, Kempe S, Müller N, Schmitz M, Bachmann M, Cartellieri M, et al: Tumor evasion from T cell surveillance. J Biomed Biotechnol 2011:918471, 2011

19. Walcott BP, Nahed BV, Mohyeldin A, Coumans JV, Kahle KT, Ferreira MJ: Chordoma: current concepts, management, and future directions. Lancet Oncol 13:e69-e76, 2012

\section{Disclosures}

The authors report no conflict of interest concerning the materials or methods used in this study or the findings specified in this paper.

\section{Author Contributions}

Conception and design: Zenonos, Fernandes Cabral, Gardner. Acquisition of data: Zenonos, Fernandes Cabral, Gardner. Analysis and interpretation of data: all authors. Drafting the article: all authors. Critically revising the article: all authors. Reviewed submitted version of manuscript: all authors. Approved the final version of the manuscript on behalf of all authors: Zenonos. Administrative/technical/material support: Fernandes Cabral. Study supervision: Zenonos, Fernandes Cabral, Fernandez-Miranda, Gardner.

\section{Correspondence}

Georgios A. Zenonos, Department of Neurological Surgery, University of Pittsburgh Medical Center, 200 Lothrop St., Ste. B400, Pittsburgh, PA 15213. email: zenonosg@upmc.edu. 